QUANTUM PHYSICS

\section{Photon lab in a circuit}

\author{
FrankK. Wilhelm and Enrique Solano
}

\section{Electrical circuits might be regarded as rather mundane pieces of classical engineering. But their electromagnetic fields are, like light, a quantum object whose energy comes in discrete units - photons.}

Both a coherent light beam and thermal, 'black-body' radiation given out by a heat source are archetypal, classical electromagnetic fields. Such fields can for most purposes satisfactorily be described as waves within the classical theory of electromagnetism formulated by James Clerk Maxwell in the 1860s. But even classical electromagnetic fields have an inherent quantum nature. That much is amply demonstrated by the experiments of Schuster et al. ${ }^{1}$, set out on page 515 of this issue, which measure the statistics of photons in microwave electromagnetic fields confined within the solidly classical surroundings of an electrical circuit.

Quantum physics owes its origin to Max Planck's postulation in 1900 of the existence of discrete quanta of energy, which were needed to formulate a consistent theory of black-body radiation. Albert Einstein, too, in his annus mirabilis of 1905 , observed that quantizing light - dividing it up into discrete packets of energy known as photons - could help to explain the photoelectric effect ${ }^{2}$. Since then, countless experiments have confirmed that electromagnetic fields are fundamentally quantized. The quantum theory of coherence, introduced by Roy Glauber in the 1960s, has provided a deeper insight into photonic systems ${ }^{3}$.

Within this framework, even classical coherent and thermal fields can be described in terms of quantum-mechanical concepts such as phase properties and photon statistics. In a coherent field, the photonic quantum state has a well-defined phase; in an incoherent, thermal field, the phase information is distributed randomly. But on the macroscopic scale - the scale of, for example, an electrical circuit - a quantum-mechanical description of electrodynamics has a fundamental credibility problem. Its probabilistic tenets simply seem irreconcilable with our everyday intuition. For that reason, quantum theories generally tend to be perceived as a theory of only the small and invisible: of photons in free space, and of atoms and molecules.

Schuster and colleagues' work ${ }^{1}$ builds on research performed on superconducting circuits, and provides crucial verification that quantum electrodynamics indeed has a role at a macroscopic scale in our everyday life. Their advance uses powerful tools, mainly developed by them earlier ${ }^{4}$, that are inspired by a pivotal innovation known as cavity quantum electrodynamics. Here, an electromagnetic field is confined inside a resonator as standing waves with discrete frequencies. This allows the study of the interaction between the quantized field and the atoms in the resonator. Major tests, such as the generation and detection of non-classical states, have been performed on electromagnetic fields of microwave wavelengths (in the range of centimetres) confined in such three-dimensional cavities ${ }^{5,6}$.

The authors' technique uses a microwave field that is not confined in three dimensions in air, as in a standard cavity, but is guided and defined in a one-dimensional, coplanar waveguide - a refined, on-chip version of the coaxial cables common in conventional electronics. According to theory ${ }^{7}$, such guided waves are quantum fields similar to light. The equivalent of a cavity is defined by interrupting the waveguide with capacitors that prevent the electromagnetic wave passing. A Cooperpair box, a tiny superconducting circuit consisting of a metallic island and a bigger electrode, plays the role of the atom with which the field interacts in a standard cavity (Fig. 2 on page 516).

The physics of this Cooper-pair box can be simplified as two quantum states that differ by a single pair of electrons on the island, and therefore in their electrical dipole moment, which is a measure of the distribution of charge. Because of the greater separation of the charges, the dipole moments involved are huge compared with those of real atoms. Added to the fact that the wave modes supported by the cavity are densely packed in small volumes, this means that the atom-field coupling energy is high. Whereas Schuster and colleagues previously demonstrated just the coherent coupling of the atom and field", they can thus now investigate the precise quantum character of the field.

The authors measure ${ }^{1}$ the associated quantum probabilities of the photon numbers in the resonator by imprinting information about the field state on the artificial atom, and then observing the atom reusing the radiation trapped in the cavity. When the artificial probe atom couples to the cavity far from the cavity's resonance frequency, the transition frequency between the atom's two quantum states is shifted - a phenomenon known as the a.c. Stark effect. If the field were completely classi$\mathrm{cal}$, this frequency shift would be constant, and proportional to the square of the electrical field. But in the quantum world, the discreteness of the field energy and the probabilistic nature of the field state allow the frequency shift to have only certain values, which are proportional to the number of photons present in the cavity (Fig. 3 on page 517). The height of the peaks corresponding to these frequency shifts reflects the probability of different numbers of photons in the cavity. If the cavity is exposed to a simulated source of thermal radiation, these probabilities follow Planck's thermal law (Fig. 4 on page 517 ). If the cavity is coherently excited, one obtains a Poisson distribution, as emerges from Glauber's theory of coherence.

For sceptics looking for possible loopholes in quantum theory, this proof of the quantum nature of the confined microwave field is the start of a new generation of tests of its non-classicality. Beyond the significance this set-up has for the foundations of quantum physics on the macroscopic scale, it opens up new perspectives in quantum measurement and its potential use in quantum information processing. The complete measurement of the quantum field - not only photon statistics, but also phase information - is a logical next step, and could be achieved by applying a powerful method known as quantum homodyne tomography. But implementing that technique in the microwave domain will require further work and fresh ideas owing to the lack of effective photodetectors for microwave light ${ }^{8}$.

These kinds of precise measurement would be easier if the field to be measured were separated from the field that does the measuring?, and the fact that they are combined is a weakness of Schuster and colleagues' current setup. The next step is to prepare and verify other genuine quantum objects, such as single microwave photons, entangled multimode fields, and single- and two-mode squeezed states. Schuster and colleagues' work ${ }^{1,4}$ is sparking many theoretical proposals on how to achieve all this, and more. With such impetus, quantum optics on a chip could soon emerge as an exciting new subdiscipline of physics.

FrankK. Wilhelm is at the Institute for Quantum Computing and Department of Physics and

Astronomy, University of Waterloo,

Ontario N2T 2V9, Canada.

Enrique Solano is in the Physics Department, ASC and CeNS, Ludwig-Maximilian-Universităt, 80333Munich, Germany, and in the Sección Fisica, Pontificia Universidad Católica del Perú, Lima, Peru.

e-mails: fwilhelm@iqc.ca;

enrique.solano@physik.Imu.de

1. Schuster, D. L etal. Nature 445,515-518 (2007).

2. Einstein, A.Ann Phys. 17,132-148 (1905).

3. Glauber, R.L. Phys Rev. 131, 2766-2788 (1963).

4. Wallraft, A. et al. Nature 431, 162-167(2004).

5. Raimond, 1-M et al. Rex Mod Phys. 73, 565-582 (2001).

6. Walther, H, Varcoe, B. T. H, Englert, B.G.\& Becker, T. Rep. Prog. Phys 69,1325-1382(2006).

7. Blais, A, Huang R-S, Wallraff, A, Girvin S, M. \& Schoelkopt, R. I. Phys. Rex A69, 062320 (2004).

8. Mariantoni, M.et al. preprint at wwwaniv.org/condmat/0509737 (2005).

9. Storcz, M.J.etal.preprint at wwweaniv.org/condmat/0612226 (2006) 